\title{
Many-body effects in the cyclotron resonance of a magnetic dot
}

\author{
Nga T. T. Nguyen ${ }^{1, *}$ and F. M. Peeters ${ }^{1,2, \dagger}$ \\ ${ }^{1}$ Departement Fysica, Universiteit Antwerpen, Groenenborgerlaan 171, B-2020 Antwerpen, Belgium \\ ${ }^{2}$ Departamento de Física, Universidade Federal do Ceará, Caixa Postal 6030, Campus do Pici, 60455-760 Fortaleza, Ceará, Brazil
}

(Received 17 April 2009; revised manuscript received 16 July 2009; published 30 September 2009)

\begin{abstract}
Intraband cyclotron resonance (CR) transitions of a two-electron quantum dot containing a single magnetic ion is investigated for different Coulomb interaction strengths and different positions of the magnetic ion. In contrast to the usual parabolic quantum dots where $\mathrm{CR}$ is independent of the number of electrons, we found here that due to the presence of the magnetic ion Kohn's theorem no longer holds and CR is different for systems with different number of electrons and different effective electron-electron Coulomb interaction strength. Many-body effects result in shifts in the transition energies and change the number of CR lines. The position of the magnetic ion inside the quantum dot affects the structure of the CR spectrum by changing the position and the number of crossings and anticrossings in the transition energies and oscillator strengths.
\end{abstract}

DOI: 10.1103/PhysRevB.80.115335

PACS number(s): 78.67.Hc, 71.55.Eq, 75.75.+a, 75.50.Pp

\section{INTRODUCTION}

Single-doped magnetic impurity quantum dots (QDs) (Ref. 1) are considered as promising nanostructures for spintronic physics. Besides, these systems allow probing the impurity spin states. ${ }^{2,3}$ Furthermore, spin information in these spin-based devices can be stored ${ }^{4}$ (and retrieved) in the magnetic moment of the $\mathrm{Mn}$ ion. In these nanosystems one can confine a small number of electrons that can be manipulated by, e.g., the gate voltage. These diluted magnetic semiconductor nanostructures have attracted a lot of attention to theoreticians and experimentalists within the past ten years. Those studies $^{2-12}$ include investigations of the electronic structure, the magnetic properties, optical absorption, etc. For a recent review of experimental and theoretical work on QDs doped with magnetic impurities the reader is referred to Ref. 13

Magnetic-optical properties of nonmagnetic few-electron QDs were investigated in, e.g., Refs. 14-19. It was found theoretically ${ }^{15,16}$ and experimentally ${ }^{17}$ that the optical cyclotron resonance (CR) energies in the case of quadratic confining potentials are independent of the number of electrons. In other words, the electron-electron $(e-e)$ interaction does not result in any observable changes in the far-infrared (FIR) spectrum for different $N_{e}$-electron systems. This theorem is no longer valid in the presence of impurities as found experimentally ${ }^{20}$ and theoretically. ${ }^{21}$

In this paper we study a magnetic semiconductor system $[\mathrm{Cd}(\mathrm{Mn}) \mathrm{Te}]$ in the presence of a Mn-ion of spin $5 / 2\left(\mathrm{Mn}^{2+}\right)$. We find that the excitation energy spectrum of the quantum dot will change due to the presence of the Mn ion. Different CR lines in the electron absorption energy spectrum are found as a consequence of the electron-magnetic-ion $(e-\mathrm{Mn})$ spin-spin-exchange interaction. Positioning the Mn-ion at different positions inside the QD affects the results significantly. We found also that the $e$-e interaction influences the electron excitation spectrum.

A two-dimensional few-particle circular parabolic QD was considered as a model system to study, e.g., the pair Coulomb interaction effect. ${ }^{22,23}$ Here we will use this model to examine the effect of the $e-e$ interaction on the intraband absorption spectrum of a two-electron QD containing a single Mn-ion. Detailed studies of the FIR spectra in the case without a $\mathrm{Mn}$ ion were done for quantum dot molecules ${ }^{19}$ containing few electrons in which the $e$-e interaction leads to small shifts in the peak position of the CR spectrum.

The intraband energy absorption spectrum of a magnetic QD was calculated by Savić et al. ${ }^{24}$ for a single electron in the presence of one and two $\mathrm{Mn}$ ions in a 3D nonparabolic CdTe/ZnTe QD system, and recently by ourselves ${ }^{25}$ for a single electron in a two-dimensional purely parabolic single-doped-Mn ${ }^{2+}$ CdTe self-assembled QD. Both studies pointed out that with the presence of the $\mathrm{Mn}$ ion, the in-plane intraband absorption energy spectra exhibits several CR lines as a consequence of the $e$-Mn interaction. Here we extend our previous work to the case of two electrons and study the effect of the $e-e$ interaction on the CR and the magnetooptical absorption spectrum. One way to see that the $e-e$ interaction will affect the CR spectrum is by writing the twoelectron Hamiltonian in terms of center-of-mass $\left(\overrightarrow{R_{c}}\right.$ $\left.=\frac{\vec{r}_{1}+\vec{r}_{2}}{2}, \vec{P}=\overrightarrow{p_{1}}+\overrightarrow{p_{2}}\right)$ and relative $\left(\vec{r}=\overrightarrow{r_{1}}-\overrightarrow{r_{2}}, \vec{p}=\overrightarrow{p_{1}}-\overrightarrow{p_{2}}\right)$ coordinates,

$$
H=H_{R_{c}}+H_{r}+H_{s M}+H_{Z},
$$

where

$$
\begin{gathered}
H_{R_{c}}=\frac{1}{2 M^{*}}\left(\vec{P}+Q \overrightarrow{A_{c}}\right)^{2}+\frac{1}{2} M^{*} \omega_{0}^{2} R_{c}^{2}, \\
H_{r}=\frac{1}{2 M^{*}}\left(\vec{p}+\frac{1}{2} Q \overrightarrow{A_{r}}\right)^{2}+\frac{1}{2} M^{*} \omega_{0}^{2}(r / 2)^{2}+\frac{K Q^{2}}{4 \epsilon r},
\end{gathered}
$$

and

$$
H_{s M}=J_{c} \vec{M} \cdot\left[\overrightarrow{s_{1}} \delta\left(\overrightarrow{R_{c}}+\frac{\vec{r}}{2}-\vec{R}\right)+\vec{s}_{2} \delta\left(\overrightarrow{R_{c}}-\frac{\vec{r}}{2}-\vec{R}\right)\right]
$$

are, respectively, the center of mass, the relative, and the electron spin $\mathrm{Mn}$-ion spin Hamiltonian. $H_{Z}$ is the total Zeeman spin energy. $M^{*}=2 m^{*}$ and $Q=2 e$ are, respectively, the total mass and total charge of the electrons. $\omega_{0}$ is the confin- 
ing frequency, $\overrightarrow{A_{c}}=\frac{1}{2}\left(\vec{B} \times \overrightarrow{R_{c}}\right)$ and $\overrightarrow{A_{r}}=\frac{1}{2}(\vec{B} \times \vec{r})$ are the centerof-mass and relative magnetic vector potential, respectively. In a $\mathrm{CR}$ experiment the long-wavelength radiation interacts only with the center of mass through $e \vec{E} \cdot \overrightarrow{R_{c}}$, where $\vec{E}$ is the electric field of the FIR. Notice that due to the interaction of the electron spin with the Mn-ion spin $\vec{M}$ the center of mass is coupled with the relative coordinates which contain information on the $e-e$ interaction.

In the next section we describe our theoretical approach to solve the many-particle system which is based on the configuration interaction (CI). Section III presents our numerical results. A summary and our conclusions are given in Sec. IV.

\section{THEORETICAL APPROACH}

The Hamiltonian ${ }^{11,12}$ for a parabolic quantum dot containing two electrons interacting with a single $\mathrm{Mn}$ ion $\left(\mathrm{Mn}^{2+}\right)$ in the presence of a perpendicular magnetic field in secondquantized form reads

$$
\begin{aligned}
\hat{H}= & \sum_{i, \sigma} E_{i, \sigma} c_{i, \sigma}^{+} c_{i, \sigma}+\frac{1}{2} \hbar \omega_{c}\left(g_{e} m^{*} / m_{0} S_{z}+g_{\mathrm{Mn}} m^{*} / m_{0} M_{z}\right) \\
& +\frac{1}{2} \sum_{i j k l \sigma \sigma^{\prime}}\left\langle i, j\left|V_{0}\right| k, l\right\rangle c_{i, \sigma^{+}}^{+} c_{j, \sigma^{\prime}}^{+} c_{k, \sigma^{\prime}} c_{l, \sigma}-\sum_{i j} \frac{1}{2} J_{i j}(\vec{R}) \\
& \times\left[\left(c_{i, \uparrow}^{+} c_{j, \uparrow}-c_{i, \downarrow}^{+} c_{j, \downarrow}\right) M_{z}+c_{i, \uparrow}^{+} c_{j, \downarrow} M^{-}+c_{i, \downarrow}^{+} c_{j, \uparrow} M^{+}\right],
\end{aligned}
$$

where $i$ in the sum is the single-particle Fock-Darwin state: $\varphi_{i=\left\{n_{r} l\right\}}(\vec{r})=1 / l_{H} \sqrt{n_{r} ! / \pi\left(n_{r}+|l|\right) !}\left(r / l_{H}\right)^{|l|} e^{-i l \theta} e^{-r^{2} / 2 l_{H}^{2}} L_{n_{r}}^{|l|}\left(r^{2} / l_{H}^{2}\right)$ having corresponding on-site energy: $E_{i, \sigma}=\hbar \omega_{H}\left(2 n_{r}+|l|+1\right)$ $-\hbar \omega_{c} l / 2$ with the frequency $\omega_{H}=\sqrt{\omega_{0}^{2}+\omega_{c}^{2} / 4}$ that defines a new length $l_{H}=\sqrt{\hbar / m^{*} \omega_{H}}$, where $\omega_{c}=e B / m^{*}$ is the cyclotron frequency. This new effective length is related to the confinement length $l_{0}$ via the relation: $l_{H}=l_{0} /\left(1+\Omega_{c}^{2} / 4\right)^{1 / 4}$ with $\Omega_{c}$ $=\omega_{c} / \omega_{0}$. It is convenient to use this dimensionless parameter $\Omega_{c}$ instead of the frequency $\omega_{c}$. For our numerical work we consider the host semiconductor CdTe and indicate the Landé $g$ factor of the electron and the Mn ion by $g_{e}$ and $g_{\mathrm{Mn}}$, respectively. The strength of the electron-Mn-ion spin-spinexchange interaction is evaluated via: $J_{i j}(\vec{R})=J_{c} \varphi_{i}^{*}(\vec{R}) \varphi_{j}(\vec{R})$ as the product of single-electron Fock-Darwin wave functions $i$ and $j$ at the position of the Mn-ion. We define a dimensionless parameter: $\lambda_{C}=l_{0} / a_{B}$ as the Coulomb interaction strength ${ }^{12}$ with $a_{B}^{*}=4 \pi \epsilon_{0} \epsilon \hbar^{2} / m^{*} e^{2}$ the effective Bohr radius.

We use the many-body wave function, built up from the Fock-Darwin basis, namely, the Slater determinant for a specific configuration of the electrons and the $\mathrm{Mn}$ ion, which now includes the spin part of the $\mathrm{Mn}$ ion $\chi_{\mathrm{s}}(\vec{M})$. As an example, the two-electron wave function for configuration $k$ $=\left\{m, n, \boldsymbol{\varsigma}_{\vec{M}}\right\}=\left\{\left(n_{r}, l, s_{z}\right)_{m},\left(n_{r}, l, s_{z}\right)_{n}, \boldsymbol{\varsigma}_{\vec{M}}\right\}$ is written,

$$
\psi_{k}\left(\overrightarrow{x_{1}^{*}}, \overrightarrow{x_{2}^{*}}, \vec{M}\right)=\frac{1}{\sqrt{2}}\left[\phi_{m}\left(\overrightarrow{x_{1}^{*}}\right) \phi_{n}\left(\overrightarrow{x_{2}^{*}}\right)-\phi_{n}\left(\overrightarrow{x_{1}^{*}}\right) \phi_{m}\left(\overrightarrow{x_{2}^{*}}\right)\right] \chi_{\varsigma}(\vec{M}),
$$

where $\overrightarrow{x_{i}^{*}}=\left(\overrightarrow{r_{i}}, \overrightarrow{s_{i}}\right)$ is the radial and spin coordinates of the $i$ th electron in state $\phi_{\alpha}\left(\vec{x}_{i}^{*}\right)=\varphi_{\alpha} \chi_{\sigma}\left(\overrightarrow{s_{i}}\right)$-a product of the Fock-
Darwin and the spin coordinate - as the single-particle wave function. Now we employ the CI method to define the wave function of the system: $\Psi\left(\overrightarrow{x_{1}^{*}}, \overrightarrow{x_{2}^{*}}, \vec{M}\right)=\sum_{k=1}^{N_{c}} c_{k} \psi_{k}\left(\overrightarrow{x_{1}^{*}}, \overrightarrow{x_{2}^{*}}, \vec{M}\right)$ as a linear combination of all possible configurations $N_{c}$, where $\psi_{k}$ is given by Eq. (4). The number of configurations $N_{c}$ is determined by the number of Fock-Darwin orbitals $N_{s}$, the number of electrons which in the present study is 2 , with taking into account a factor of 2 due to the electron spin $1 / 2$ and a factor of 6 due to the Mn-ion spin size 5/2: $N_{c}$ $=6 \times\left(\begin{array}{c}2 \\ 2 N_{s}\end{array}\right)$. For our numerical work we use the set of parameters used in Refs. 11 and 12 with $m^{*}=0.106 m_{0}, g_{e}=-1.67$, $g_{\mathrm{Mn}}=2.02, a_{B}^{*}=52.9 \AA, J_{c}=1.5 \times 10^{3} \mathrm{meV}^{2}$, and dielectric constant $\epsilon=10.6$. The effective Coulomb interaction strength $\lambda_{C}$ is changed by changing $l_{0}$, i.e., which is a measure for the size of the QD, which is typically about tens of angstroms. $N_{c}$ is taken sufficiently large to guarantee convergency.

\section{NUMERICAL RESULTS}

\section{A. Cyclotron resonance}

The oscillator strength for circular polarized light is given by

$$
f_{i j}=\frac{2 \Delta E_{i j}}{\hbar \omega_{H}} \cdot \frac{\left|A_{i j}\right|^{2}}{l_{H}^{2}},
$$

with the transition amplitude for our two-electron system,

$$
A_{i j}=\sum_{p=1}^{N_{e}=2}\left\langle\Psi_{i}\left(\vec{r}_{1}, \vec{r}_{2}\right) \mid r_{p} e^{ \pm i \theta_{p} \mid} \Psi_{j}\left(\vec{r}_{1}, \vec{r}_{2}\right)\right\rangle
$$

The \pm sign refers to right and left circular polarized light. $\Delta E_{i j}=E_{j}-E_{i}$ is the cyclotron transition energy for the system from state $i$ to state $j$. Clearly, the spins of the electrons and the $\mathrm{Mn}$ ion are not involved in the transition. From expression (6), we see that one of the electrons can transit to a higher (lower) energy level while the other electron can be involved in another transition. Therefore, the wave function part of the other electron can be separated from each term of the Slater determinant while one-electron resonates.

We express the Slater determinant (4) in the language of permutations for identical particles: $\psi_{k}\left(\overrightarrow{x_{1}^{*}}, \overrightarrow{x_{2}^{*}}, \vec{M}\right)$ $=(2 !)^{-1 / 2} \chi_{\mathrm{s}}(\vec{M}) \Sigma_{P} \delta_{P} \hat{P} \phi_{m}\left(\overrightarrow{x_{1}^{*}}\right) \phi_{n}\left(\overrightarrow{x_{2}^{*}}\right)$, where $\hat{P}$ is one of the $2 !$ permutation operators. $\delta_{P}$ equals +1 for symmetric and -1 for antisymmetric case. The quantum number $m$ [e.g., $m$ $\left.=\left(n_{r}, l, s_{z}\right)\right]$ and $n$ (for electron two) characterize the twoelectron configuration $\{m, n\}$, and the operator $\hat{P}$ acts on the order of these single-electron configurations.

Integrating the right-hand side of Eq. (6) over $\vec{r}_{1}$ and $\vec{r}_{2}$, we end up with

$$
\begin{aligned}
& A_{i j}=(2 !)^{-1} \delta_{M_{z}^{i}, M_{z}^{j}} \sum_{P} \sum_{P^{\prime}} \delta_{P} \delta_{P^{\prime}} \hat{P} \widehat{P^{\prime}}\left\{\sum_{\alpha}^{N_{C}} \sum_{\alpha^{\prime}}^{N_{C}} \delta_{n, n^{\prime}} c_{\alpha}^{*} c_{\alpha^{\prime}}\right.
\end{aligned}
$$

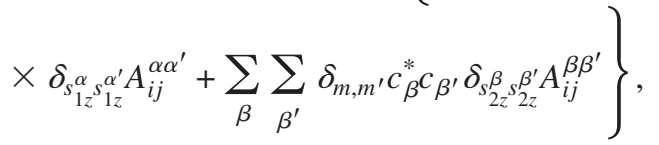

where $\alpha(\in P)$ and $\alpha^{\prime}\left(\in P^{\prime}\right)$ stand for one electron and 


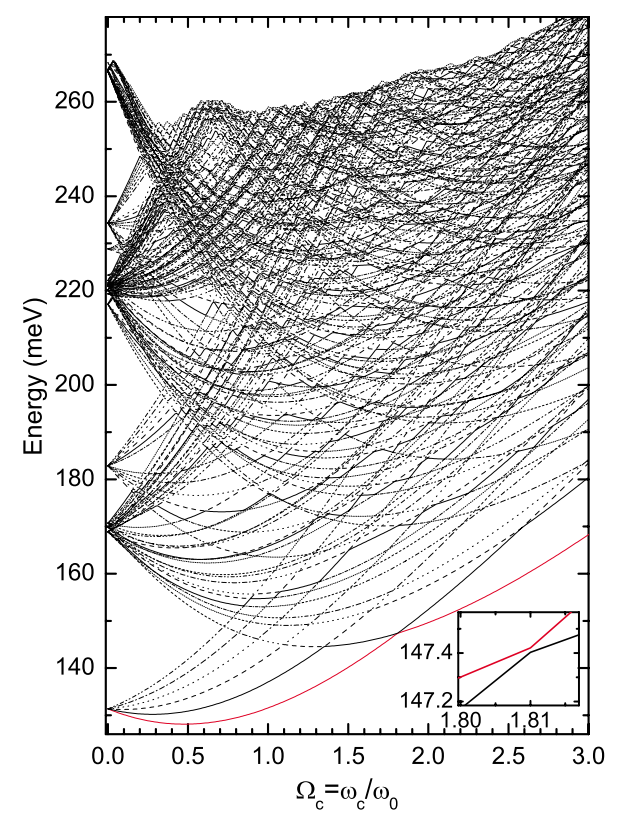

FIG. 1. (Color online) First 200 energy levels of the spectrum of a two-electron-magnetic QD with the $\mathrm{Mn}$ ion located at the center of the QD and for a Coulomb interaction strength $\lambda_{C}=0.5$. The inset is the anticrossing point of the first two energy levels at $\Omega_{c}=1.81$.

$\beta(\in P)$ and $\beta^{\prime}\left(\in P^{\prime}\right)$ for the other electron. The prime indicates the final state $j$. The matrix element $A_{i j}^{\alpha \alpha^{\prime}}$

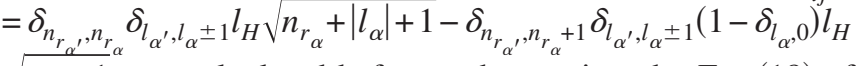
$\sqrt{n_{r_{\alpha}}+1}$ was calculated before and was given by Eq. (18) of Ref. 18 .

We use the following Lorentzian broadened formula for the different CR peaks:

$$
\sigma_{i}(E)=\sum_{j} \frac{\Gamma_{i j}}{\pi} \cdot \frac{f_{i j}}{\left(E-E_{i j}\right)^{2}+\Gamma_{i j}^{2}},
$$

where $i$ and $j$ refer to the initial and final states, respectively. In the following results we concentrate on the transitions where the initial state is the ground state (GS) and we will identify $\sigma_{1}(E)$ by $\sigma(E) . \Gamma_{i j}$ is the broadening parameter that is taken to be about $0.1-1 \mathrm{meV}$ in our numerical calculations.

We first calculate the oscillator strength (OS) for the case without and with the presence of the $\mathrm{Mn}$ ion, taking into account all allowed transitions of the two electrons. The system with the Mn-ion located at the center of the dot has the energy spectrum as shown in Fig. 1 when the effective Coulomb interaction strength is $\lambda_{C}=0.5$. Many crossings and anticrossings are found in the spectrum that were not present in the case without a Mn ion. The anticrossings, which are a consequence of intermixing of higher quantum states due to the presence of the Mn ion, lead to energy gaps between the levels, and result in unusual behaviors in the cyclotron resonance spectrum.

For $N_{e}=2$, the pure ferromagnetic (FM) phase, where the two electrons have spins parallel to the spin of the Mn ion, does not exist. This is opposite to the case for $N_{e}=1$, which is a consequence of the closed $s$ orbital for $N_{e}=2$. This re-

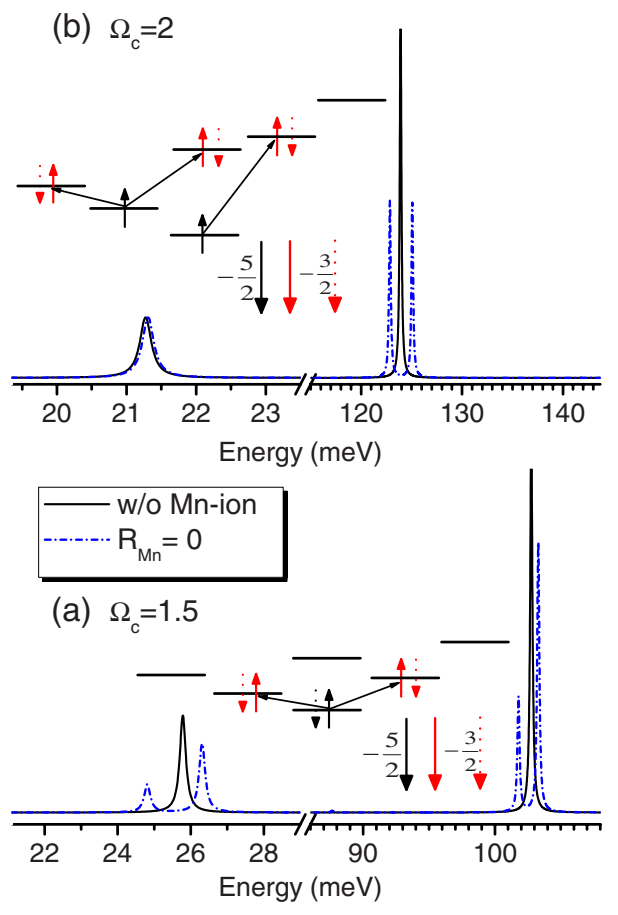

FIG. 2. (Color online) $\sigma(E)$ vs energy for two different magnetic fields where the system is in the (a) FM and in the (b) AFM state for a two-electron QD with (blue dash-dotted curve) and without (black solid curve) the presence of a Mn-ion for $\lambda_{C}=0.5$. The Mn ion is positioned in the center of the QD. Two schematic plots in (a) and (b) describe the corresponding transitions in which black is for GS and red for excited state; solid is for main contribution and dotted for minor contribution to the total OS.

sults in zero diagonal elements for the $e$-Mn exchange matrix. The neighboring off-diagonal elements that describe the spin exchange of the electrons with the Mn ion of the configurations with different $S_{z}$ and $M_{z}$ but satisfying $S_{z}+M_{z}$ $=$ const., which are in general very small, now turn to be the main contributions to the exchange interaction energy. Therefore, a weakened "FM" state is still found with a total spin slightly larger than zero. The magnitude of these offdiagonal contributions is small and depends on the position of the magnetic ion. Moving the Mn ion to other positions inside the QD when the system is still in the FM phase can affect the structure of the energy spectrum of the system. With increasing the magnetic field, the system will transit to a phase where the two electrons will have spin up and are antiferromagnetically attracted to the $\mathrm{Mn}$ ion. This phase is called antiferromagnetic (AFM). The FM-AFM transition can be seen from the inset of Fig. 1 through the anticrossing between the GS and the second level at $\Omega_{c}=1.81$. The Mn ion is always "frozen" with spin projection $-5 / 2$ in case of nonzero magnetic field.

Figures 2(a) and 2(b) are the magneto-optical absorption spectrum $\sigma(E)$ for two values of the magnetic field $\Omega_{c}=1.5$ (FM) and $\Omega_{c}=2(\mathrm{AFM})$, respectively, for the system in case without (black solid) and with (blue dash-dot) the presence of the Mn ion (at the center of the dot). Two schematic diagrams describe possible electron transitions with OS exceeding $0.1 \%$ of the total OS taking place within the $s, p$, and $d$ shells. When the system is in the FM phase, the main tran- 
sitions are such that the final state has the quantum numbers $\left(n_{r}, L_{z}, S_{z}, M_{z}\right)=(0,1,0,-5 / 2)$ (the second peak) or $(0$, $-1,0,-5 / 2)$ (the fourth peak) as the major configuration and $(0,1,-1,-3 / 2)$ (the first peak) or $(0,-1,-1,-3 / 2)$ (the third peak) as the minor configuration, respectively. We recall ${ }^{25}$ that the number of CR lines in the case of a single-electron quantum dot doped with a Mn ion when the system is in the FM state is two and the $e$ (spin-down)-Mn-ion (spin-down) interaction affects the CR spectrum in this state through shifting the cyclotron energy and/or the presence of crossings. The spin-exchange interaction becomes stronger when the system is in the AFM state (electron and Mn-ion spin antiparallel), resulting in the presence of more CR lines. However, the major CR lines in this case are two. Let us go back to the current system when it is in the AFM state where we find three major CR lines as can be seen in Fig. 2(b). The first peak is due to an electron transition from the $p^{+}\left[\left(n_{r}, l\right)\right.$ $=(0,1)]$ orbital to the $d^{+}(0,2)$ orbital corresponding to the resonance from the GS with major configuration $(0,1,1$, $-5 / 2)$ to the final state with major configuration $(0,2,1$, $-5 / 2$ ). For the other two pronounced peaks, the one that appears at the smaller transition energy is for the $s$-electron transition to the $p^{-}(0,-1)$ and its oscillator strength is slightly larger than the other peak that stands for the electron transition from the $p^{+}(0,1)$ orbital to the $d^{0}(1,0)$ orbital. Their final states have as dominant configurations $(0,0,1$, $-5 / 2)$ and $(1,0,1,-5 / 2)$, respectively. The CR transitions that become allowed due to spin exchange have a much smaller oscillator strength than the major transitions and can be neglected. For the case without a $\mathrm{Mn}$ ion, the system transits from the state $\left(n_{r}, L_{z}, S_{z}\right)=(0,0,0)$ to the state $(0,1,1)$. Note that if one keeps increasing the magnetic field, the electrons will occupy higher quantum states resulting in the GS wave function having quantum numbers, e.g., $(0,3,1)$ or $(1,1,1)$, and so on. Consequently, the number of possible $\mathrm{CR}$ lines increases.

Here, we will discuss in more detail the transitions resulting from the $(0,1,1)$ GS. Note that only two of the three allowed CR lines are observable, namely, the $p^{+}$electron to the $d^{+}$[the first solid peak in Fig. 2(b)] and the $s$ electron and the $p^{+}$electron to, respectively, the $p^{-}$and the $d^{0}$ orbital that have the same OS and transition energy [the second solid peak in Fig. 2(b)]. These two coinciding CR lines split in the presence of the Mn ion as can be seen by the dash-dotted curve where the system is in the AFM state. We note that for $\Omega_{c}=2$ [Fig. 2(b)] the first peak corresponding to the transition of the $p^{+}$electron to the $d^{+}$orbital has almost the same transition energy in case without and with the Mn ion. This is due to the zero exchange interaction energy between the $s$ and the $p$ orbitals when the Mn ion is located at the center of the dot. The energy difference between these two peaks and the number of spin-exchange CR lines will increase as one moves the Mn ion away from the center of the QD. We will come back to this point in a later discussion.

The OS and absorption energy spectrum of the above case over a wide range of magnetic field are found in Figs. 3 and 4, respectively. For the case without a Mn ion, these quantities are, respectively, reviewed in the insets (b) and (c) of Fig. 3 and in the inset (b) of Fig. 4. Figure 4(b) is identical to the case of one-electron QD due to the $N_{e}$ independence of

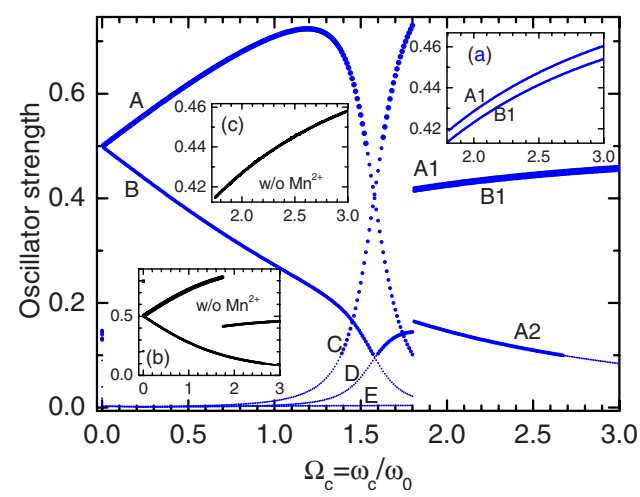

FIG. 3. (Color online) Oscillator strength of a two-electron QD with the $\mathrm{Mn}$ ion located at the center of the $\mathrm{QD}$, (a) magnification of the region of $1.8 \leq \Omega_{c} \leq 3$ where the system transits to the AFM phase. (b) the OS of the same QD without a Mn ion and (c) the magnification of the same region as in (a) but for the same situation as in (b). The thickness of the curve is proportional to the value of the OS. A, B, C, D, and E label the five branches when the system is in the FM and $\mathrm{A} 1, \mathrm{~A} 2$, and $\mathrm{B} 1$ label the branches when the system is in the AFM phase.

the absorption spectrum for parabolic confinement without a $\mathrm{Mn}$ ion. The thickness of the transition absorption energy curves plotted in Fig. 4 is proportional to the OS whose values are plotted in Fig. 3. As we can see from these two plots, the CR transitions for the case when the Mn ion is present is very different from the case without a Mn ion. For the magnetic field region where the system is in the FM phase $\Omega_{c} \leq 1.81$, the differences are significant which can be seen from the presence of the two crossings in the OS in Fig. 3 that correspond to the two anticrossings in the transition energy in Fig. 4. At the FM-AFM transition point, there are discontinuities. For the AFM region, the difference is in the

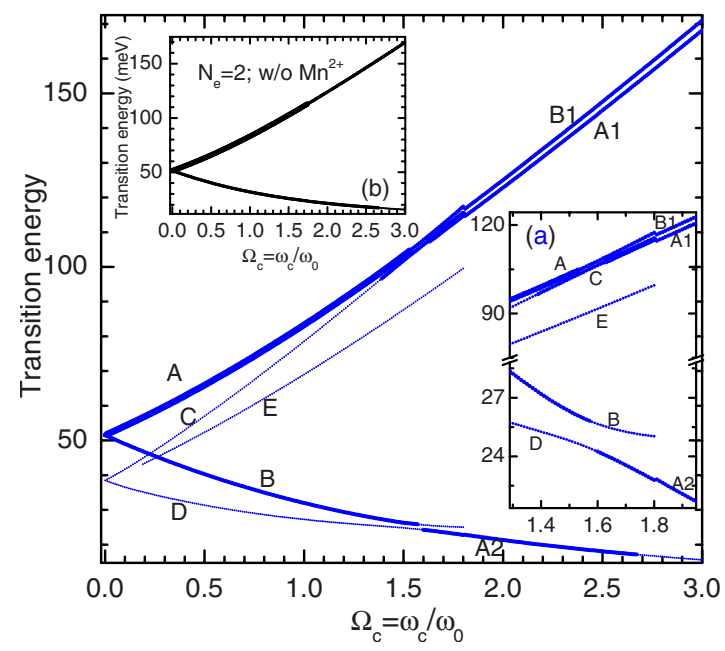

FIG. 4. (Color online) Transition energy corresponding to the OS of Fig. 3 and for the case without a Mn ion [inset (b)]. (a) Magnification of the transition energy of the main plot for the magnetic field region $1.3 \leq \Omega_{c} \leq 2.0$. The thickness of the curve is proportional to the respective OS value and the labels corresponding to the transition energy branches of the respective OS ones plotted in Fig. 3. 
separation of the two lines that was not present in the case without a Mn ion as shown, respectively, in Figs. 3(a) and 4(a) and Figs. 3(b) and 4(b). Note that the discontinuity in the OS of Fig. 3(b) is due to the fact that the CR spectrum transits from two to three lines, where the last two CR lines have the same transition energy (they are the transitions $s$ $\rightarrow p^{-}$and $p^{+} \rightarrow d^{0}$ ). If these OS are added, there would be no discontinuity in Fig. 3(b). Let us first discuss the branches of the OS for the region $\Omega_{c}<1.4$ (before the first crossing) which we name region I. In this region, the system is in the FM phase. The two electrons mostly stay in the $s$ shell with antiparallel spins. The transitions are from the GS to the two $p$ orbitals with high OS. The latter are the two higher branches in the OS curve plotted in Fig. 3. The final states, which now mix with several quantum states, include, respectively, $(0,-1,0,-5 / 2)$ (higher) or $(0,1,0,-5 / 2)$ as their main contribution. We see also two lower branches that are the curves corresponding to the electron transitions from the GS to the two $p$ orbitals but with different spin states. The final states are now such that the electron that is excited to a higher state will flip its spin which is compensated by a change of the Mn-ion spin. We found that the final states corresponding to these $\mathrm{CR}$ lines have, respectively, the quantum numbers $(0,-1,-1,-3 / 2)$ (higher) and $(0,1,-1,-3 / 2)$ as their major contribution. This is understood via the $e$-Mn-ion spin-spin-exchange interaction. These two curves have a smaller OS than the above two due to the small contribution of the configuration $(0,0,-1,-3 / 2)$ in the GS while the major one is $(0,0,0,-5 / 2)$.

Now we will discuss the results for the higher magnetic field region. At the first crossing, $\Omega_{c}=1.45$, we observe the exchange between two branches as seen in Fig. 3 . The higher branch of the two lower branches is higher in energy than the lower branch of the two higher branches in region I of the OS plot. This is the case up to the next two crossings at $\Omega_{c}=1.58$. The region $1.45 \leq \Omega_{c} \leq 1.58$ is named region II. Within this magnetic field region, the final state which has the configuration $(0,-1,-1,-3 / 2)$ as its major contribution to the OS becomes larger in OS as compared to the state which has the configuration $(0,1,0,-5 / 2)$. At $\Omega_{c}=1.58$ we find two crossings with an exchange of OS. This is a consequence of the fact that the energy spectrum exhibits crossings (and anticrossings) of energy levels. Next we consider the AFM phase, $\Omega_{c} \geq 1.81$, and focus on the two higher branches that stay very close to each other. Remember that for the case without a Mn ion, these branches are degenerate [as can be seen from Figs. 3(c) and 4(b)]. In the presence of the $\mathrm{Mn}$ ion, this degeneracy is lifted and their OS differ about $1 \%$ of the total OS and their energy differs by $3-4 \mathrm{meV}$ or about $6.8 \%$ of the confinement energy $\hbar \omega_{0}$. They correspond to transitions of the spin-up electron from the $s$ shell to the $p^{-}$orbital (lower branch) and of the spin-up electron from the $p^{+}$orbital to the $d^{0}$ orbital as discussed before in Fig. 2, respectively. For these transitions, the Mnion spin with $M_{z}=-5 / 2$ is unaltered. The other lower branch corresponds to the transition of the $p^{+}$electron to the $d^{+}$ orbital. The anticrossings in the transition energy are illustrated in Fig. 4(a) which magnifies the region $1.3 \leq \Omega_{c}$ $\leq 2.0$.

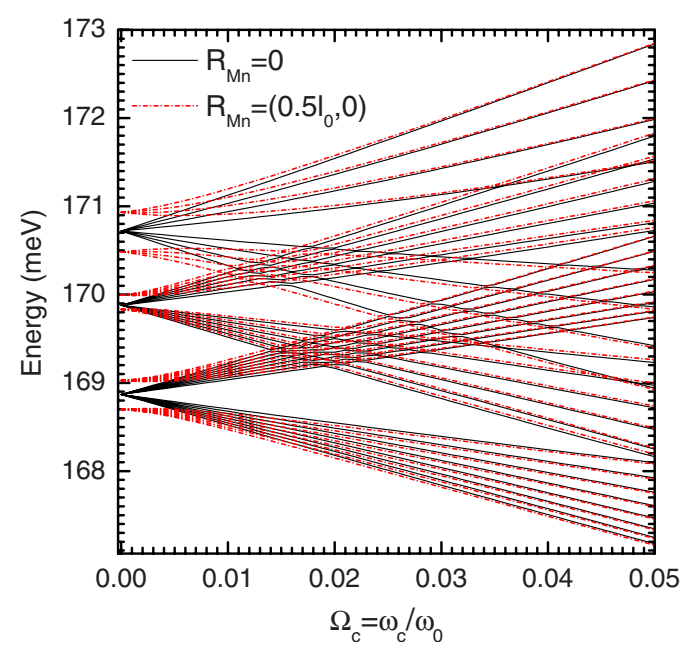

FIG. 5. (Color online) 36 energy levels (from level 7 to level 42 that are grouped into 8-8-6-6-4-4 lines) of a two-electron QD with the $\mathrm{Mn}$ ion located at the center or at $\left(0.5 l_{0}, 0\right)$ of the QD with the Coulomb interaction strength $\lambda_{C}=0.5$.

\section{B. Mn-ion position dependence of the intraband excitation spectrum}

Moving the $\mathrm{Mn}$ ion to another location inside the QD affects the energy spectrum as shown in Fig. 5, where we focus on the small magnetic field region. The black solid lines and the red dashed lines are the 36 energy levels starting from level 7 for the cases that the $\mathrm{Mn}$ ion is located at the center and at $\left(0.5 l_{0}, 0\right)$, respectively. We first notice that the $B=0$ GS energy in both cases has the same degeneracy (i.e., $6)$. For the higher energy levels different degeneracies are found; for $R_{\mathrm{Mn}}=0$ we have 16-12-8 while for the other case we have 8-8-6-6-4-4 fold degeneracy. When applying a magnetic field, these degeneracies are further lifted due to the Zeeman effect. We note that, e.g., for the two eightfolddegenerate levels [in the case that the Mn-ion is located at $\left(0.5 l_{0}, 0\right)$-the red dash-dotted curves] or the two 16-folddegenerate levels (in the case that the Mn ion is located at the center of the dot-the black solid curves), as the magnetic field increases, the lower energy level corresponds to states with positive total azimuthal quantum number, in this case +1 - one electron in the $s$ shell and the other in the $p^{+}$orbital and the higher energy level has negative total azimuthal quantum number -1 -one electron in the $s$ shell and the other in the $p^{-}$orbital, etc. The degeneracy of the higher energy levels for $B=0$, i.e., 8,6 , or 4 , etc., comes from the ferromagnetic (e.g., $\vec{M}+\vec{S}=\overrightarrow{7 / 2}$ for the case of eightfold degeneracy) or antiferromagnetic (e.g., $\vec{M}+\vec{S}=\overrightarrow{5 / 2}$ for the case of sixfold degeneracy) coupling of the two electrons with the Mn ion. Note that all the 36 energy levels in Fig. 5 refer to the $s$ and $p$ orbitals. At higher magnetic fields, the energy spectrum exhibits further differences when $R_{\mathrm{Mn}}$ is varied in, e.g., the number and the positions of the crossings and anticrossings, the energy gaps of the anticrossings, etc.

As we discussed above, the system that consists of two electrons and a single $\mathrm{Mn}$ ion does not exhibit a very clear FM phase since the state where the two electrons stay in the $s$ shell with antiparallel spins minimizes the GS. The energy 
gained by the direct antiferromagnetic coupling of the Mn ion to the spin-up electron is balanced by the ferromagnetic exchange with the other spin-down electron. This holds as long as the two electrons occupy the $s$ orbital. Therefore, the FM-AFM transition magnetic field is almost not changed when we vary the position of the $\mathrm{Mn}$ ion. For example, the FM-AFM takes place at $\Omega_{c}=1.81$ for the cases that the $\mathrm{Mn}$ ion is located at the center and at $\left(0.5 l_{0}, 0\right)$ and at $\Omega_{c}=1.79$ for the case the $\mathrm{Mn}$ ion is located at $\left(l_{0}, 0\right)$. To see the Mnion position dependence on the CR spectrum, we will focus on the high magnetic field region where the system is in the AFM phase with both electrons having spin up and one of them accommodating a higher orbital, e.g., the $p^{+}$orbital. The allowed electron transitions now are the three lines that start from the GS to the $p^{-}$(for the $s$ electron) and to the $d^{+}$ and $d^{0}$ orbitals (for the $p$ electron). Note that for the case that the $\mathrm{Mn}$ ion is located at the center of the dot, the exchange interactions between the $s$ and $p$ orbitals are zero, while these terms increase as the Mn-ion is moved away from the center, e.g., to $\left(0.5 l_{0}, 0\right)$ or $\left(l_{0}, 0\right)$. Consequently, the behavior of the OS changes. The first transition that corresponds to the transition of the $p^{+}$electron to the $d^{+}$orbital is shifted. The other two transitions that stay very close in energy and correspond to the other transitions of the $s$ electron and the $p^{+}$electron to the $p^{-}$and $d^{0}$ orbitals [the solid lines at the right-hand side in the diagram in Fig. 2(b)], respectively, are most separated for the case the Mn ion is located at the center of the QD. The reason is that for $R_{\mathrm{Mn}}=0$, only the exchange interactions between the $s$ and the $d^{0}$ orbitals are nonzero (and equal) within the involved shells $s, p$, and $d$. This means that the final state with the configuration of the $s$ electron and the $d^{0}$ electron as their main contribution to the wave function is more enhanced in energy. This difference leads to a larger separation between the two CR lines for $R_{\mathrm{Mn}}=0$. Qualitatively, this separation is about 3-4 meV for $R_{\mathrm{Mn}}=0$ and $0.9-1 \mathrm{meV}$ for $R_{\mathrm{Mn}}=0.5 l_{0}, 0.5 \mathrm{meV}$ for $R_{\mathrm{Mn}}=l_{0}$ and almost zero for $R_{\mathrm{Mn}}$ $=2 l_{0}$. In the last case the problem converts to the problem without a $\mathrm{Mn}$ ion. The two transitions merge to a single one as seen before [black curve in Fig. 2(b)].

We complete this subsection by investigating the absorption spectrum of the system for $R_{\mathrm{Mn}}=0.5 l_{0}$ in a range of magnetic field $\Omega_{c}=(1.5,2.5)$ that includes the FM-AFM transition [see Fig. 6]. We focus our discussions on the two transitions discussed in the previous paragraph that correspond to the right-hand side CR lines in Fig. 2(b). As the system transits to the AFM phase, the "correlations" between the $s$ and $p, s$ and $d, p$ and $d$, etc. become nonzero for almost all values of the coupling strength. Consequently, the final states of the two transitions gain energy from the exchange interaction part. Their difference reduces, leading to the fact that these two peaks stay closer in energy as compared to the case that the $\mathrm{Mn}$ ion is located at the center of the dot. However, the stronger peak (at smaller transition energy) which corresponds to the transition of the $s$ electron when the system is in the AFM phase can become more pronounced or smaller than the other transition as illustrated in Fig. 6. As the system transits to the AFM phase $\left(\Omega_{c} \geq 1.9\right)$, we see that within the magnetic field range $\Omega_{c}=1.9-2.1$ the peak that corresponds to the transition of the $p^{+}$electron to the $d^{0}$ orbital is higher (having a larger OS) as compared to

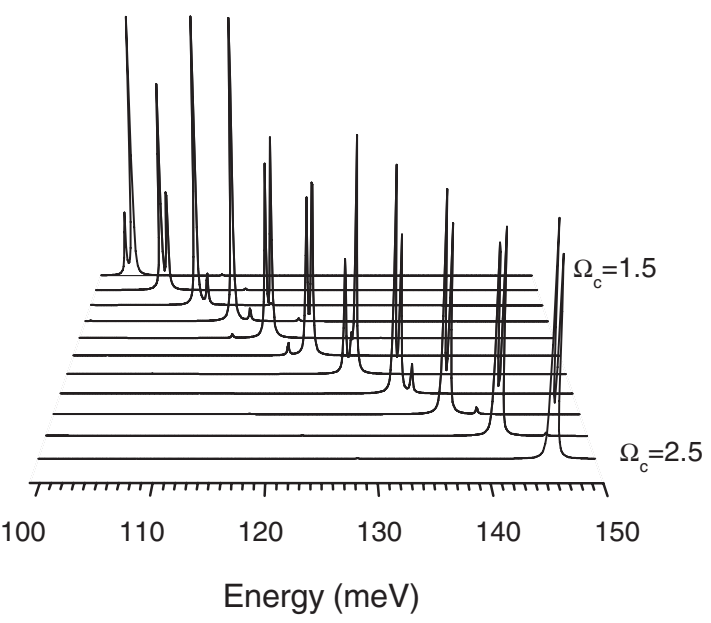

FIG. 6. Magneto-optical absorption spectrum focused on the two higher transitions for the same QD as plotted in Fig. 4 for the case the $\mathrm{Mn}$ ion is located at $\left(0.5 l_{0}, 0\right)$. The magnetic field range is $(1.5,2.5)$ with steps of 0.1 .

the other peak that corresponds to the transition of the $s$ electron to the $p^{-}$orbital. Within the magnetic field where $\Omega_{c}=2.2-2.3$ the OS of the two peaks exchange. This exchange happens again at $\Omega_{c}=2.4$ and we obtain the last exchange in this figure for $\Omega_{c}=2.5$. Each time that there is an OS exchange between these two peaks there is a crossing in the OS (and an anticrossing) in the transition energy. When the $\mathrm{Mn}^{2+}$ is displaced to $\left(0.5 l_{0}, 0\right)$, the difference in the OS value between these two peaks is now larger, but the difference in energy is smaller. Note that also smaller peaks appear next to the main ones. The small peak for $\Omega_{c}=1.5$ is a transition from the GS with dominant configuration $(0,0,0$, $-5 / 2)$, which has $(0,0,-1,-3 / 2)$ as a minor contribution, to the state with the configuration $(0,-1,-1,-3 / 2)$ as its main contribution to the wave function. For $\Omega_{c}=1.9$ the small peak is due to the transition to the state with the configuration $(0,-1,-1,-3 / 2)$ as its main contribution.

\section{Electron-electron interaction effect}

Here we investigate how the strength of the $e-e$ interaction influences the $\mathrm{CR}$ absorption spectrum. Its influence on the first 24 energy levels is plotted in Fig. 7 in case without $e-e$ interaction (solid black curves) and two different $\lambda_{C}[0.2$ (blue dash-dot-dotted curves) and 0.5 (violet dash curves)]. Crossings and anticrossings of these energy levels occur at different magnetic fields (the smaller $\lambda_{C}$ the larger the crossing field $\Omega_{c}$ ).

Lets turn to the transition energy and for reference purposes we turn off the Coulomb interaction strength (see Fig. 8). Without the Coulomb interaction, the FM-AFM transition takes place at a larger magnetic field $\Omega_{c}=3.44$ as compared to $\Omega_{c}=1.81$ for the case with $e$-e interaction (see Fig. 4 and Ref. 12). There are generally six CR lines (as compared to four for the case with $e-e$ interaction) with $\mathrm{OS}$ exceeding $0.1 \%$ of the total OS [Fig. 8(b)]. The main difference is that the dominant $\mathrm{CR}$ lines [conventional transitions, e.g., from the GS with the quantum state $(0,0,0,-5 / 2)$ as the main 


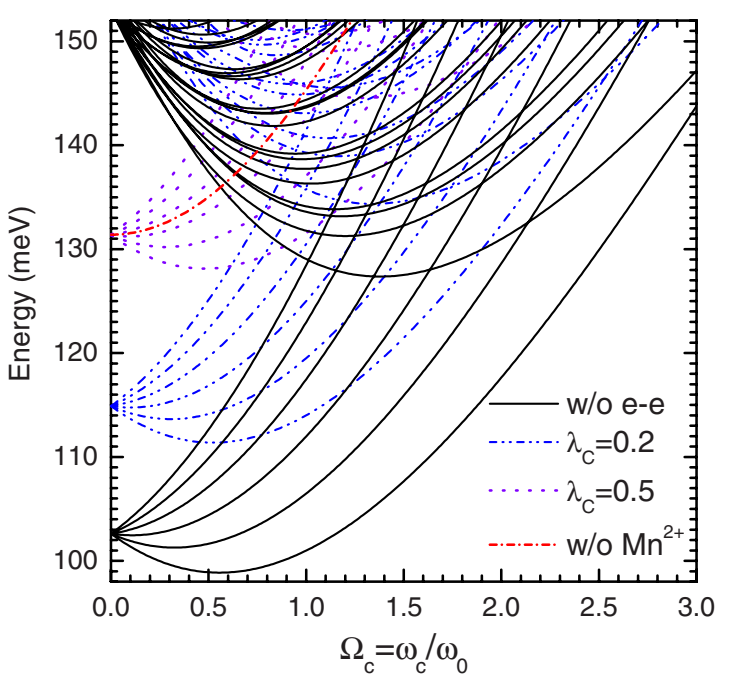

FIG. 7. (Color online) Energy spectrum plotted for the first 24 levels for several different Coulomb interaction strengths: without $e$-e interaction, $\lambda_{C}=0.2,0.5$. Red dash-dotted curve is the energy of the same $\mathrm{QD}\left(\lambda_{C}=0.5\right)$ without a $\mathrm{Mn}$ ion for reference. The energy spectrum for the case $\lambda_{C}=0.2$ is scaled to the case of $\lambda_{C}=0.5$.

configuration to the final state with the quantum state $(0, \pm 1,0,-5 / 2)]$ do not have a much larger OS than the secondary transitions-the transitions appearing as a consequence of the $e-\mathrm{Mn}$-ion exchange interactions, as can be seen from Figs. 3 and $8(b)$ ( $\leq 15 \%$ of the total OS). These six lines correspond to transitions of the electrons from the GS containing the major quantum state $\left(n_{r}, L_{z}, S_{z}, M_{z}\right)$, to the six final states that contain their dominant contributions to the wave functions as combinations of the following four quantum states: $\quad\left(n_{r}, L_{z}+1, S_{z}, M_{z}\right), \quad\left(n_{r}, L_{z}+1, S_{z}-1, M_{z}+1\right)$, $\left(n_{r}, L_{z}-1, S_{z}, M_{z}+1\right)$, and $\left(n_{r}, L_{z}-1, S_{z}-1, M_{z}+1\right)$. Depending on the value of the applied magnetic field, the relative contributions of these states to the wave function will change, leading to crossings as seen at $\Omega_{c}=0.13,0.14$ in the inset of Fig. 8(b). If we increase the magnetic field further the CR spectrum collapses into four CR lines. The lines that are the results of the exchange $e-\mathrm{Mn}$-ion interaction (C and F) become close in energy to the "conventional" lines (B and E). This behavior is not seen in case of interacting electrons. The reason is that when the Coulomb interaction is turned on, see Fig. 4, the $e$-Mn spin-spin interaction is weakened by the Coulomb repulsion. Note that the two additional peaks in the CR spectrum are a consequence of the breaking of the selection rule by the $e$-Mn exchange interaction.

When comparing the low magnetic field behavior (see Figs. 3 and 4) of the CR spectrum we note a very different behavior when we turn off the $e$-e interaction (see Fig. 8). To understand this different behavior we plot in Fig. 9(a) the magneto-optical absorption spectrum for the system with a Mn-ion located at the center of the dot and $\lambda_{C}=0.2$ for the magnetic field range $0<\Omega_{c}<3$. As compared to the system studied in Figs. 3 and 4 with $\lambda_{c}=0.5$ : first, the AFM phase takes place at a larger magnetic field $\left(\Omega_{c}=2.53\right.$ as compared to 1.81); second, the low OS branches survive up to higher fields. The FM-AFM transition occurs at $\Omega_{c}=1.24$ and 0.96, for $\lambda_{C}=1$ and 1.5 , respectively. As the $e$-e interaction
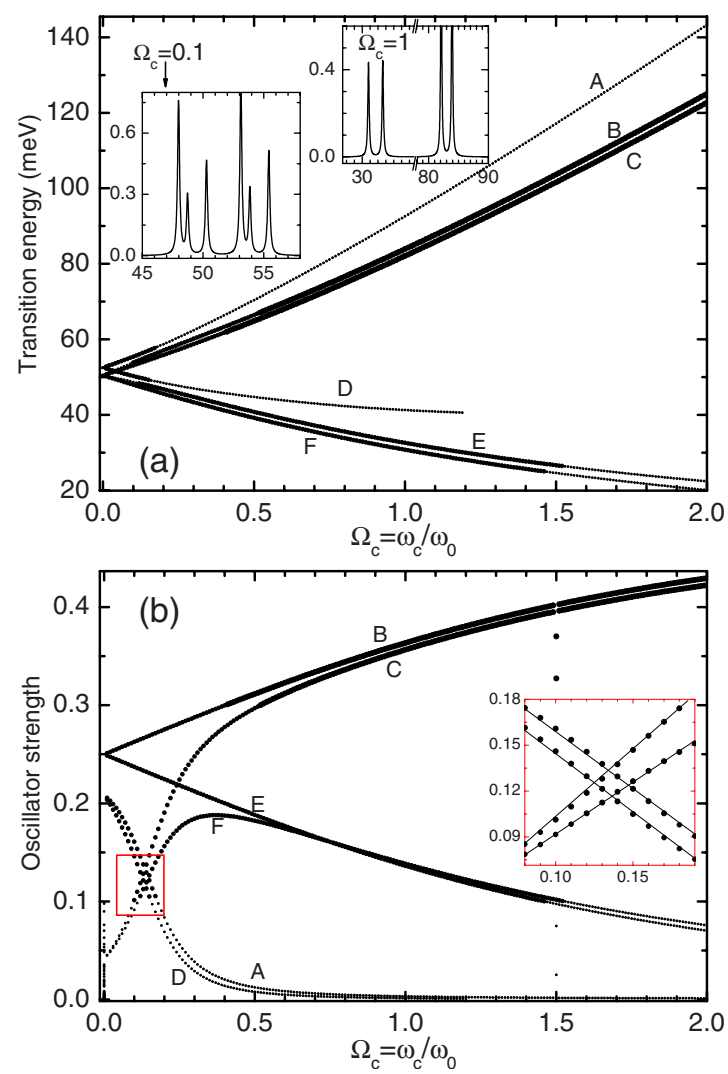

FIG. 8. (Color online) Transition energy (a) and OS (b) of a two-electron $\mathrm{QD}$ without $e$ - $e$ interaction for the case the Mn ion is at the center of the dot. The thickness of the curve is proportional to the OS value (up to $\Omega_{c}=2$, there is still no FM-AFM transition happening). The two insets in (a) are the $\sigma(E)$ plotted for two magnetic fields: $\Omega_{c}=0.1$ and 1 . A, B, C, D, E, and F label the six branches of the transition energy in (a) and the respective OS branches in (b). The inset in (b) is a magnification of the red rectangular where we use four lines to connect the data points of the four corresponding CR lines.

strength increases, e.g., from $\lambda_{C}=0.2$ [see Fig. 9(a)] to $\lambda_{C}$ $=2$ [see Fig. 9(b)], the low OS branches that appear due to the spin exchange of the electrons and the $\mathrm{Mn}$ ion contribute less to the total OS. In Fig. 9(a), the contribution of the exchange terms to the total OS stays appreciable within the magnetic field range $(0,1.7)(\mathrm{FM})$. The low OS branches appear on both "sides" of the transitions of the $s$ electrons: to the $p^{+}$(left-lower transition energy) and to the $p^{-}$(righthigher transition energy) orbitals [see the lower peaks of Fig. 9(a)], meaning that the final states with the configurations having $\left(0,0, S_{z}+M_{z}=-5 / 2\right)$ as the main contribution have nonzero OS. While these low OS branches appear only on the "side" of the $p^{+}$electron resonating to the $d^{+}$orbital within the magnetic field region $(1.4,1.7)$ (AFM), see the red rectangular in Fig. 9(b). It means that only the final state with the main quantum configuration as $(0,2,0,-3 / 2)$ has a nonzero contribution (as a minor) to the oscillator strength, while there is no gain from the exchange interactions during the transitions of the $p^{+}$electron and the $s$ electron to the $d^{0}$ and $p^{-}$orbitals, respectively. However, in any case, the exchange interactions always separate these two transitions in 


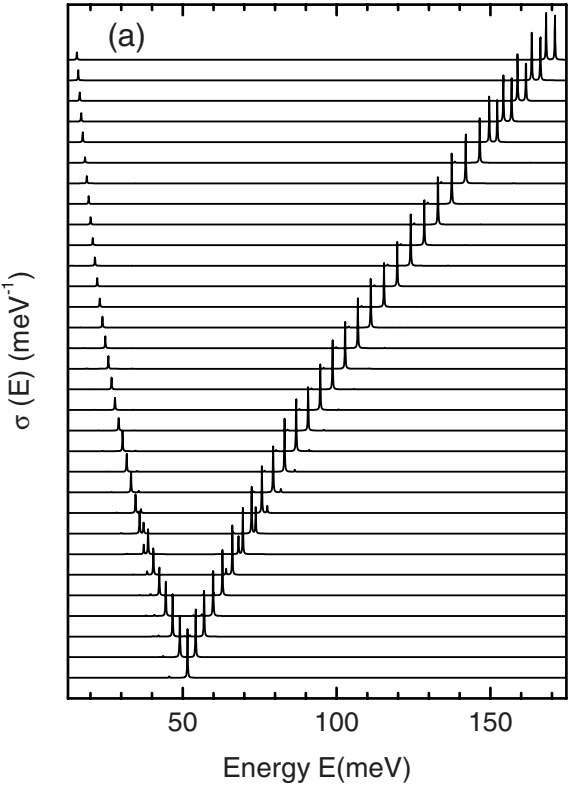

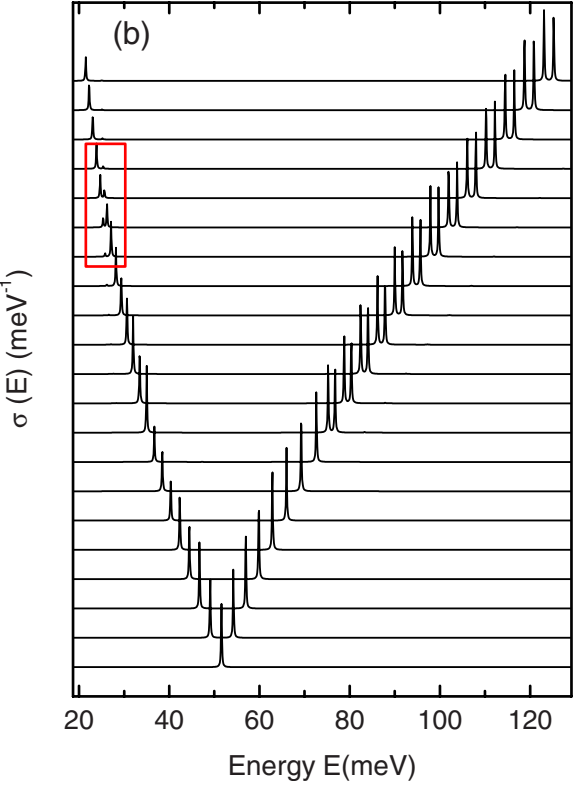

FIG. 9. (Color online) Magneto-optical absorption spectrum scaled to the case of $\lambda_{C}$ $=0.5$ obtained for the case the Mn ion is located at the center of the QD for $\lambda_{C}=0.2$ (a) and $\lambda_{C}=2$ (b). Results are shown in the magnetic field range $(0,3)(a)$ and $(0,2)(b)$ with steps of 0.1 . energy (about $6.8 \%$ of $\hbar \omega_{0}$ for $R_{\mathrm{Mn}}=0$ ), as can be observed in Figs. 9(a) and 9(b), for magnetic field $\Omega_{c} \geq 2.53$ and $\Omega_{c}$ $\geq 0.79$ for $\lambda_{C}=0.2$ and 2 , respectively.

Last, we study the combined effects of the position of the $\mathrm{Mn}$ ion and the Coulomb interaction strength on the CR absorption spectrum. Due to the exchange interactions between all included quantum orbitals, the transitions of the $s$ electron to the $p^{-}$orbital and of the $p^{+}$electron to the $d^{0}$ orbital have different energies depending on the strength of the Coulomb interaction as can be seen in Fig. 10. We plot $\sigma(E)$ as a function of energy for three different $\lambda_{C}(=0.2,0.5$, and 1) at $\Omega_{c}=2.6$ (AFM phase) with the Mn ion located at $\left(0.5 l_{0}, 0\right)=(13.2 \AA, 0)$. For the smallest considered $\lambda_{C}$ (i.e., $0.2)$ the two electrons are more strongly confined and the location of the $\mathrm{Mn}$ ion in this case is almost out of the effective region of the electrons. Consequently, the separation

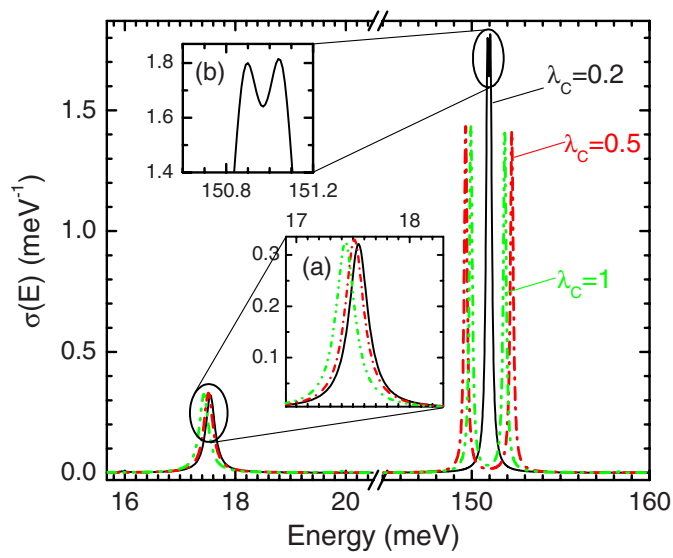

FIG. 10. (Color online) Magneto-optical absorption spectrum scaled to the case of $\lambda_{C}=0.5$ obtained for the case the Mn ion is located at $\left(0.5 l_{0}, 0\right)$ for three different Coulomb interaction strengths $\lambda_{C}=0.2,0.5$, and 1 and magnetic field $\Omega_{c}=2.6$. (a) magnification of the first major peak. We focused on the three main transitions in each case with inset (b) the magnification to see the two upper peaks for the case of $\lambda_{C}=0.2$. between the two transitions of the electrons in two different orbitals becomes very small as can be seen from the black solid curve in Fig. 10 and its inset. The first transition, the transition of the $p^{+}$electron to the $d^{+}$orbital, slightly changes as $\lambda_{C}$ changes as shown in Fig. 10(a).

\section{SUMMARY AND CONCLUSIONS}

We find that the electron transition energy in parabolic quantum dot with a single $\mathrm{Mn}$ ion depends on the strength of the $e-e$ interaction and the number of electrons in the QD. The $e-e$ interaction shifts and splits the two main branches (the upper-energy ones) of the absorption spectrum at the FM-AFM transition magnetic field. The strength of the $e-e$ interaction changes the position of the low OS branches significantly. Different branches appear with changing Coulomb interaction strength.

In a many-electron quantum dot without a $\mathrm{Mn}$ ion the $\mathrm{CR}$ spectrum consists of two peaks due to Kohn's theorem. The reason for the breakdown of Kohn's theorem is the presence of the Mn ion, which leads to spin-exchange interaction with the electrons of the QD. Moving this Mn ion to different positions in the QD corresponds to changing the magnitude of the exchange interactions and leads to changes in the absorption energy spectrum.

In the FM region, the spin-spin $e$-Mn-ion interactions share their contributions to the total OS with the two "direct" transitions of the two $s$ electrons in which the total $S_{z}$ is conserved. In the AFM region, this leads to discontinuities in the OS and the respective transition energy and separate the two transitions of the $s$ and the $p^{+}$electrons in energy. These two transitions address, respectively, the $p^{-}$and $d^{0}$ orbitals. At the FM-AFM transition magnetic field, we observed that the OS exhibits a discontinuity for all the CR peaks. When the system is in the FM phase, the transition energy exhibits only minor differences as compared to the case without a Mn ion. When the system is in the AFM phase, however, there are major differences in the absorption energy spectrum. The 
separation of the two upper peaks in the CR spectrum depends on the effective $e$-e interaction strength and the position of the Mn ion. The heights of these two upper peaks change with changing position of the $\mathrm{Mn}$ ion and their relative heights exchange OS at each anticrossing in the transition energy. The electrons spin-spin exchange with the Mn ion and the strength of it depends on the position of the $\mathrm{Mn}$ ion. Therefore, changing the Coulomb interaction strength will affect the absorption energy spectrum by, e.g., separating or merging the two above electron transitions. When the Mn ion is moved away from the center of the dot, the exchange interactions become nonzero, resulting in additional $\mathrm{CR}$ peaks. The number of these branches reduces as the Coulomb interaction strength increases. When the $e-e$ interaction is turned off, we found that the transitions that become allowed due to the spin exchanges increase in OS.

In short, in the FM phase (i.e., low magnetic field situation) the number of CR lines is increased to four for $N_{e}=2$, which compares to two lines when $N_{e}=1$. In the AFM phase (i.e., high magnetic field) the number of CR lines with substantial OS is reduced to three, while for $N_{e}=1$ it was four of which only two had an OS appreciable different from zero. These changes in the CR spectrum should be observable in a $\mathrm{CR}$ experiment. The number of electrons in the quantum dot can be varied through the application of a gate potential. In order to resolve the CR lines that are very close to each other it is recommended to use single dot spectroscopy. ${ }^{2-4}$

\section{ACKNOWLEDGMENTS}

This work was supported by FWO-Vl (Flemish Science Foundation), the EU Network of Excellence: SANDiE, the Brazilian science foundation CNPq, and the Belgian Science Policy (IAP).

\footnotetext{
*nga.nguyen@ua.ac.be

†rancois.peeters@ua.ac.be

${ }^{1}$ J. K. Furdyna, J. Appl. Phys. 64, R29 (1988).

${ }^{2}$ L. Besombes, Y. Léger, L. Maingault, D. Ferrand, H. Mariette, and J. Cibert, Phys. Rev. Lett. 93, 207403 (2004).

${ }^{3}$ Y. Léger, L. Besombes, J. Fernández-Rossier, L. Maingault, and H. Mariette, Phys. Rev. Lett. 97, 107401 (2006); L. Maingault, L. Besombes, Y. Léger, H. Mariette, and C. Bougerol, Phys. Status Solidi C 3, 3992 (2006).

${ }^{4}$ C. Le Gall, L. Besombes, H. Boukari, R. Kolodka, J. Cibert, and H. Mariette, Phys. Rev. Lett. 102, 127402 (2009).

${ }^{5}$ A. O. Govorov, Phys. Rev. B 70, 035321 (2004).

${ }^{6}$ T. Schmidt, M. Scheibner, L. Worschech, A. Forchel, T. Slobodskyy, and L. W. Molenkamp, J. Appl. Phys. 100, 123109 (2006).

${ }^{7}$ P. Wojnar, J. Suffczyński, K. Kowalik, A. Golnik, G. Karczewski, and J. Kossut, Phys. Rev. B 75, 155301 (2007).

${ }^{8}$ J. Fernández-Rossier and R. Aguado, Phys. Rev. Lett. 98, 106805 (2007); J. Fernández-Rossier, Phys. Rev. B 73, 045301 (2006).

${ }^{9}$ K. Chang, J. B. Xia, and F. M. Peeters, Appl. Phys. Lett. 82, 2661 (2003).

${ }^{10}$ M. M. Glazov, E. L. Ivchenko, L. Besombes, Y. Léger, L. Maingault, and H. Mariette, Phys. Rev. B 75, 205313 (2007).

${ }^{11}$ F. Qu and P. Hawrylak, Phys. Rev. Lett. 95, 217206 (2005).
}

${ }^{12}$ N. T. T. Nguyen and F. M. Peeters, Phys. Rev. B 78, 045321 (2008).

${ }^{13}$ A. O. Govorov, C. R. Phys. 9, 857 (2008).

${ }^{14}$ W. Kohn and J. M. Luttinger, Phys. Rev. 96, 529 (1954); J. M. Luttinger, ibid. 102, 1030 (1956).

${ }^{15}$ W. Kohn, Phys. Rev. 123, 1242 (1961).

${ }^{16}$ F. M. Peeters, Phys. Rev. B 42, 1486 (1990).

${ }^{17}$ Ch. Sikorski and U. Merkt, Surf. Sci. 229, 282 (1990).

${ }^{18}$ F. Geerinckx, F. M. Peeters, and J. T. Devreese, J. Appl. Phys. 68, 3435 (1990).

${ }^{19}$ M. Helle, A. Harju, and R. M. Nieminen, New J. Phys. 8, 27 (2006).

${ }^{20}$ R. J. Nicholas, M. A. Hopkins, D. J. Barnes, M. A. Brummell, H. Sigg, D. Heitmann, K. Ensslin, J. J. Harris, C. T. Foxon, and G. Weimann, Phys. Rev. B 39, 10955 (1989); J. Richter, H. Sigg, K. v. Klitzing, and K. Ploog, ibid. 39, 6268 (1989).

${ }^{21}$ U. Merkt, Phys. Rev. Lett. 76, 1134 (1996).

${ }^{22}$ P. A. Maksym and T. Chakraborty, Phys. Rev. Lett. 65, 108 (1990).

${ }^{23}$ A. Wojs and P. Hawrylak, Phys. Rev. B 53, 10841 (1996).

${ }^{24}$ I. Savić and N. Vukmirović, Phys. Rev. B 76, 245307 (2007).

${ }^{25}$ N. T. T. Nguyen and F. M. Peeters, Phys. Rev. B 78, 245311 (2008). 